\title{
La capilla musical del Real Colegio-Seminario del Corpus Christi en la segunda mitad del siglo XVII: biografía crítica, legado musical y magisterio de José Hinojosa (fl. 1662-†1673)
}

\author{
Autor: Dr. Rafael Sánchez Mombiedro. \\ Director: Dr. Antonio Ezquerro Esteban. \\ Institución: Universidad Politécnica de Valencia. \\ Tribunal: Dra. María Sanhuesa Fonseca (Universidad de Oviedo), Dr. Paulino Capdepón \\ Verdú (Universidad de Castilla la Mancha) y Dr. Josep Maria Salisi i Clos (Sociedad \\ Catalana de Musicología).
}

Calificación: Sobresaliente cum laude.

Fecha de defensa: 28 octubre de 2016.

\section{Resumen:}

Esta tesis está organizada en dos volúmenes. El primero (Estudio) contiene una introducción, el estado de la cuestión y los cuatro capítulos de que consta el trabajo, así como las conclusiones, la bibliografía y documentación empleadas y los índices de figuras, de tablas y onomástico. El segundo (Edición musical) contiene los criterios de edición y transcripción musicales, las notas y la edición crítica de ocho obras del compositor José Hinojosa.

En el Capitulo I se estudian los aspectos más relevantes, tanto político-económicos, como culturales y musicales, que son de utilidad para contextualizar el estudio que se desarrolla en los siguientes capítulos, como son: un análisis de la estructura social de la Valencia del siglo XVII, el fenómeno de la expulsión de los moriscos, decisivo en el devenir económico y social de la época, los sistemas de explotación agraria, la situación del clero, la corriente del inmaculismo, las manifestaciones artísticas y sus personajes más relevantes. Asimismo, se repasa la actividad de las capillas musicales, tanto las privadas como las religiosas, prestando especial atención a la prestigiosa catedral de Valencia, en la que brillaron maestros de renombre, como Juan Bautista Comes, Vicente García Velcaire, 


\section{TESIS DOCTORALES}

Diego Pontac, Urbán de Vargas, Gracián Babán, Antonio Teodoro Ortells y el organista Juan Bautista Cabanilles.

El Capitulo II está centrado en el Real Colegio-Seminario del Corpus Christi, comenzando por la figura de su fundador, Juan de Ribera (*Sevilla, 1532; †Valencia, 1611). Su formación en Salamanca y su posición, a la temprana edad de 36 años, como arzobispo de Valencia, así como el nombramiento como virrey de Valencia, le permitió abordar un proyecto deseado desde décadas atrás: la formación de nueva institución que atendiera a las claras directrices tridentinas referentes a la reforma del clero y la formación de nuevos sacerdotes. En el capítulo se detalla la gestación y creación de este Colegio-Seminario, supervisadas en todo momento por su fundador. La doble advocación cristológica -con el nombre de Corpus Christi = Eucaristía, símbolo católico, contrarreformista y diferenciador respecto a otras confesiones cristianas- y mariana, condicionó muchos factores, tanto litúrgicos como simbólicos, que incidirían en la vida de la institución.

Ribera pudo regir el Colegio-Seminario sin ataduras ni restricciones, para lo cual invirtió gran parte de su patrimonio. Contrató al pintor italiano Bartolomé Matarana, a quien encargó los frescos del cimborrio y de los muros de la iglesia, convirtiendo todo el conjunto pictórico en un programa iconográfico completo que reflejaba una a una las directrices de Trento. Varios lienzos de pintores de renombre -Francisco Ribalta, Vasco Pereira y un largo etcétera- y la construcción de dos órganos en el coro, completaron artísticamente su gran proyecto, que se convirtió en la construcción valenciana de referencia en el siglo XVII. Como colofón, invitó al rey Felipe III a presidir la inauguración, que tuvo lugar el 08.02.1604.

A pesar de toda la inversión y expectativas iniciales, los primeros años de la institución fueron complicados, si bien a partir de 1640 la economía del Colegio pudo recuperarse. Este era gobernado por seis colegiales primeros, controlados por una visita foránea, formada por los representantes religiosos y políticos más sobresalientes de la ciudad.

La capilla musical fue planeada por Ribera de forma ambiciosa: formada por setenta y ocho miembros, según indicaban sus Constituciones, los cargos y funciones estaban bien delimitados, con un régimen estricto. Los salarios quedaban estipulados en función del cargo, con cantidades suficientes para mantener la motivación entre sus miembros. El ceremonial litúrgico estaba establecido en torno a tres ejes: el Santísimo Sacramento, la Virgen y los santos y las reliquias. Un número de sesenta y nueve festividades de cierta importancia a lo largo del calendario litúrgico, exigía el concurso de una capilla amplia, nutrida y perfectamente organizada.

Ribera designó a Narciso Leysa como primer maestro titular, quien se encargó inicialmente de seleccionar al personal y de dotar a la capilla de un conjunto suficiente de materiales musicales con que poner en marcha el proyecto. En 1608, Juan Bautista Comes fue designado teniente de maestro, ejerciendo como compositor y ayudando en las tareas de dirección de la capilla. Comes, tras su paso por la capilla de la catedral de la ciudad y la 


\section{TESIS Doctorales}

Capilla Real, volvió al Corpus Christi en 1628, hasta que abandonó la dirección musical de la capilla en 1632. Desde la inauguración de la misma (1608) hasta esa fecha, se habían sucedido maestros titulares e interinos, con períodos breves e inestables, probablemente fruto de la propia inestabilidad de la institución. Entre 1632 y 1662 se encargó de la dirección un maestro interino, Marcos Pérez. En estas décadas, la capilla fue incrementando paulatinamente su patrimonio musical, reuniendo quince volúmenes nuevos, seis de ellos manuscritos y nueve impresos, con la mejor polifonía, mucha de ella impresa en Venecia y Roma, desde el epicentro del catolicismo.

El Capítulo III está dedicado íntegramente al compositor objeto de estudio, José Hinojosa (fl. 1662-†Valencia, 1673). En primer lugar, se realiza una biografía crítica del autor, que comenzó como maestro en Calatayud, hasta que en 1658 se ocupó de la dirección de la catedral de Teruel. En ella, Hinojosa disponía de un número reducido de efectivos vocales e instrumentales, a juzgar por las escasas obras conservadas del mismo.

El Colegio de Corpus Christi, tras treinta años de interinidad, se decidió a convocar oposiciones a maestro de capilla, convocatoria a la que acudió el maestro Hinojosa. A pesar de que el fallo del jurado nombró ganador de la oposición a Luis Vicente Gargallo, a la sazón maestro de la catedral de Huesca, los superiores, inesperadamente y sin razón alguna aparente, otorgaron la plaza a José Hinojosa, que rigió la capilla once años, desde 1662 hasta su fallecimiento el 30.12.1673.

El inventario de obras de Hinojosa conservadas en el archivo musical del Patriarca recoge un total de cuarenta y siete composiciones, provenientes de tres fuentes: el Libro de Partituras titulado Rayados de Musica del M[aestr]o Joseph Hinojosa-E-VAcp, Mus/CM-LP-24, r. 3978-, los dos borradores copiados por José Conejos Ortells - E-VAcp, Mus/CM-LP-18, r. 3979; CM-LP-19, r. 3980- y la música de Hinojosa conservada "a papeles".

Hinojosa compuso fundamentalmente salmos (veinticuatro), seguido de cánticos (ocho). Abundan asimismo sus composiciones para Completas (veinticinco), número incluso mayor que el de Vísperas (diecisiete), consolidando la tendencia manifestada desde su antecesor, Marcos Pérez, a propósito de solemnizar las Completas de los jueves "con toda pausa y solemnidad", como así indicaba el fundador.

El libro de Rayados de Musica de Hinojosa -CM-LP-24- fue copiado antes de 1675, con la finalidad principal de conservar su música de Completas (veintiuna de las veintidós composiciones son propias de esta hora canónica). Se trata de uno de los tres libros que conserva la capilla con obras de un solo maestro, junto a los otros dos, que recogen obras de Juan Bautista Comes y Máximo Ríos, respectivamente. En los borradores copiados por José Conejos en el siglo XVIII -CM-LP-18; CM-LP-19- se conservan ocho composiciones del maestro turolense, todas ellas de Completas. Por otra parte, se conservan multitud de recibos de copias de papeles de obras de Hinojosa existentes desde su defunción (1673) hasta la finalización del siglo XVII, lo que es indicativo del interés por preservar probablemente en activo- estos materiales por parte de la capilla. 


\section{TESIS DOCTORALES}

Hinojosa, a su llegada en 1662, se encontró con una capilla cuyas dimensiones no se acercaban a lo prescrito por el fundador en las Constituciones, si bien las funciones en el altar y en el coro podían cubrirse día a día con normalidad. Muchos capellanes segundos tenían más habilidades que las mínimas exigidas en las Constituciones (en ellas solo se indicaba que debían dominar el cantollano), lo que pudo aprovechar Hinojosa para aumentar el grueso del coro "de la capilla".

Se tiene constancia, en la época, de la existencia y uso frecuente de cuatro órganos, dos situados en el coro y dos portativos, por lo que estos pudieron utilizarse conjuntamente en algunas obras policorales de Hinojosa o de otros maestros.

Entre los infantes, destacó especialmente Antonio Teodoro Ortells, que se formó musicalmente con Marcos Pérez y José Hinojosa. Ortells dirigió la capilla tras la defunción de este, y en 1677 fue nombrado maestro de la catedral de Valencia, cargo que ocupó hasta su muerte en 1706.

En todos estos años se mantuvieron seis ministriles a cargo del maestro de capilla, con una copla formada por dos bajones, dos cornetas, un sacabuche y una arpa, lo que, sumado a los efectivos anteriores, formaba un grupo que fluctuaba entre veintiséis y treinta y un ejecutantes, cifras considerables que, aunque no llegaban a las pretensiones del fundador, pueden considerarse suficientes para el trabajo diario y actuación al completo en los grandes eventos litúrgicos.

En el Capitulo IV se realiza un exhaustivo estudio de ocho de las obras de Hinojosa, seleccionadas según criterios de representatividad y variedad. Las obras analizadas muestran que: 1/ Hinojosa utilizaba textos y géneros/formas de grandes dimensiones, que solemnizaban con esplendor y magnificencia los momentos más importantes del calendario litúrgico; 2/ El tratamiento de la policoralidad por parte de este autor, muestra cierta originalidad y un estadio más plenamente barroco, con la utilización de 12 voces distribuidas en hasta 4 ó 6 coros, y son representativas de un incipiente concertato vocal, como preludio del concertato instrumental más propio del siguiente siglo; 3/ Las obras de Hinojosa muestran un tratamiento exhaustivo de las proporciones entre las secciones que las componen, con una buena equidistancia entre las partes; 4/ Hinojosa conocía lo indicado por los tratadistas a propósito de la composición de determinados géneros/formas, como misas, cánticos y salmos, aunque en ocasiones "huía" de los cánones establecidos, en la búsqueda de un estilo creativo personal y adaptado a las necesidades litúrgicas; 5/ sus composiciones son un buen modelo del uso, transformación y reelaboración del material temático empleado en varias de sus partes, lo que les confiere un carácter cíclico y unitario, y muestran el buen oficio de Hinojosa como compositor; 6/ este aprovechaba los recursos teatrales de la época, con el uso y combinación de los diferentes coros y los espacios escénicos que estos ocupaban en el templo del Corpus Christi, un efecto muy "a lo barroco", que cumplía a la perfección su papel de "catequización" de los asistentes a las celebraciones; 7/ Hinojosa utilizaba con asiduidad figuras retórico-musicales y recursos de word painting (bipotyposis o descripción de objetos), 


\section{TESIS DOCTORALES}

siempre asociados al texto que acompañaban, con el fin de potenciar su significado; 8/ la ubicación de los coros en diferentes ángulos del templo, unido al uso de instrumentos acompañantes singulares, como la tiorba o el archilaúd, ofrecía un juego de contrastes espectacular, de volumen, timbre y proyección del sonido proveniente desde lo alto, que conseguía impactar a los fieles y atraer su atención; 9/ por último, los materiales musicales examinados muestran una interesante perdurabilidad, lo que denota el interés de la capilla por continuar interpretando la música de Hinojosa y, a su vez, la capacidad de "resistencia" de unas composiciones que seguían aprovechándose para el acompañamiento litúrgico.

Para completar este estudio, se ha comparado el uso de determinadas figuras retórico-musicales en las obras de Hinojosa y otras similares encontradas en obras de compositores europeos de referencia del siglo XVII, tanto de forma sincrónica (con composiciones de las décadas de 1660 y 1670) como diacrónica (incluyendo obras entre 30 y 40 años inmediatamente anteriores e inmediatamente posteriores al período trabajado), lo que permite situar a Hinojosa y su obra en un marco más amplio -aproximadamente el comprendido entre 1620 y 1710-, y ofrece un panorama lo suficientemente representativo y diversificado.

Con todo ello, y tras la transcripción y el estudio pormenorizado de una parte representativa de su repertorio, se ha podido enmarcar su obra en un amplio contexto europeo, lo que permite ser valorada y apreciada en su justa medida. 